\title{
How localised are energy dissipation processes in nanoscale interactions?
}

Sergio Santos ${ }^{1}$, Victor Barcons ${ }^{2}$, Albert Verdaguer ${ }^{3}$, Josep Font ${ }^{2}$, Neil H Thomson ${ }^{4}$, Matteo Chiesa $^{1,5}$

\footnotetext{
${ }^{1}$ Laboratory of Energy and Nanosciences, Masdar Institute of Science and Technology, Abu Dhabi, UAE ${ }^{2}$ Departament de Disseny i Programació de Sistemes Electrònics, UPC Universitat Politècnica de Catalunya Av. Bases, 61, 08242 Manresa, Spain ${ }^{3}$ Centre d' Investigació en Nanociència i Nanotecnologia (CIN2) (CSIC-ICN), Esfera UAB, Campus de la UAB, Edifici CM-7, 08193-Bellaterra, Catalunya, Spain ${ }^{4}$ School of Physics and Astronomy, University of Leeds, LS2 9JT, UK.

${ }^{5}$ Dep. of Mechanical Engineering, Massachusetts Institute of Technology, 77 Massachusetts
} Avenue Cambridge, MA 02139-4307

\begin{abstract}
We describe fundamental energy dissipation in dynamic nanoscale processes in terms of the localization of the interactions. In this respect, the areal density of the energy dissipated and the effective area of interaction in which each process occurs are calculated for four elementary dissipative processes. It is the ratio between these two, which we term $\mathrm{M}$ that provides information about how localized the interactions are. We show that neither the phase lag, nor the magnitude of the energy dissipated alone provide information about energy localization but $\mathrm{M}$ has to be considered instead.
\end{abstract}

Energy dissipation, nanoscale processes, viscosity, hysteresis, atomic force microscopy 


\section{Introduction}

The increasing interest in Nanotechnology arises from the fact that nanoscale processes and properties lie in between the realm of atoms and that of the macroscale. Thus, the nanoscale can be seen as an interface between the laws governing atomic processes and the everyday macroscale world where Newton's laws apply [1-2]. Research in this area has led to the discovery of graphene [3-5], fullerene and nanotube molecules with true nanoscale dimensions[6], artificial atoms such as quantum dots and the understanding of processes in the domain of molecular physics leading to fields ranging from molecular electronics[1-2], to the control of enzymatic and catalytic processes which have made possible, amongst others, the advent of genomics[7] and nanofabrication with biological molecules[8-10].

With the Atomic Force Microscope (AFM), the interaction between a tip of nanoscale dimension and planar surfaces, quantum dots and biomolecules can be monitored. Thus this instrument provides a means to quantify specific phenomena occurring in a very small volume of interaction[11-12]. Furthermore, because of its versatility, the AFM has rapidly evolved from the contact mode [13] (CM AFM), where the tip is in continuous repulsive contact with the surface, to dynamic modes[14-15] (dAFM), where the cantilever is vibrated in the proximity of the surface, and other modes such as magnetic, thermal and conductive AFM modes [2]. Nevertheless, while it has been over 13 years since the first analytical expression [16] to determine the energy dissipated per cycle in the dynamic modes was provided, the details on the specific mechanisms of dissipative processes are still emerging [17-23]. In a significant contribution Garcia et al. showed that the derivative of the energy dissipated per cycle with decreasing 
oscillation amplitude can be used to identify short range viscosity and long range hysteresis [19].

Here, we discuss elementary dissipative processes in dynamic nanoscale interactions in terms of the density of the energy dissipated and how localized dissipative processes are. We show that these depend not only on the magnitude of the dissipative force component, but on a combination between the nature of the dissipative process, the effective area (radius) of interaction, which we term S (r), and the dynamics at the nanoscale junction. In this contribution, we calculate $\mathrm{S}$ for each dissipative process, the density of energy dissipated per cycle or $\rho$ and the ratio between the two. This provides a means to quantify energy distribution functions in the nanoscale and predict or tune the level of energy localization in the interaction. Moreover, we identify S as the source of phase contrast resolution. By analyzing each dissipative process, we show that each of them contributes independently to phase contrast formation. Furthermore, each dissipative process presents a characteristic degree of localization contributing to the total phase contrast as a superposition of all. Nevertheless, neither the phase contrast, nor $\rho$ are sufficient criteria to understand how localized the dissipative processes are. In this respect, we define a parameter $M$ as a ratio of $\rho$ and $S$. This ratio shows that negligible phase lag and energy dissipation may result from highly localized dissipative processes and vice versa. We account for contact (c) and non-contact (nc) interactions, where these terms are used throughout with this meaning. In particular, we model $\mathrm{S}$ in the contact region due to attractive and repulsive interaction forces with areas $S_{a}$ and $S_{r}$ respectively and the area $S_{n c}$. corresponding to the attractive forces in the nc region. 
(a)

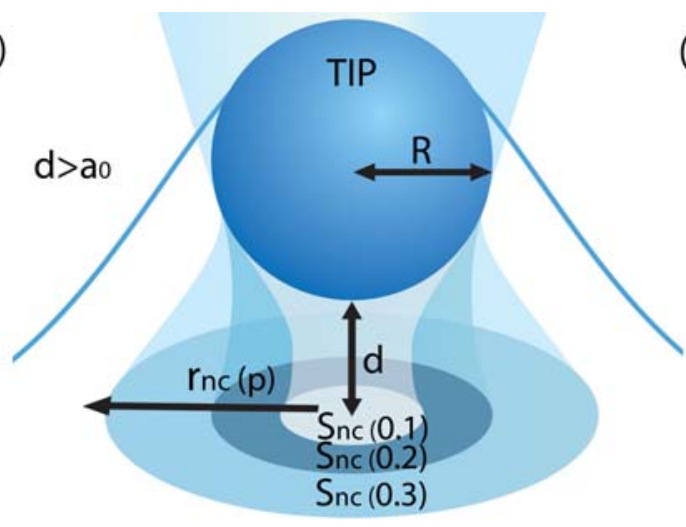

(c)

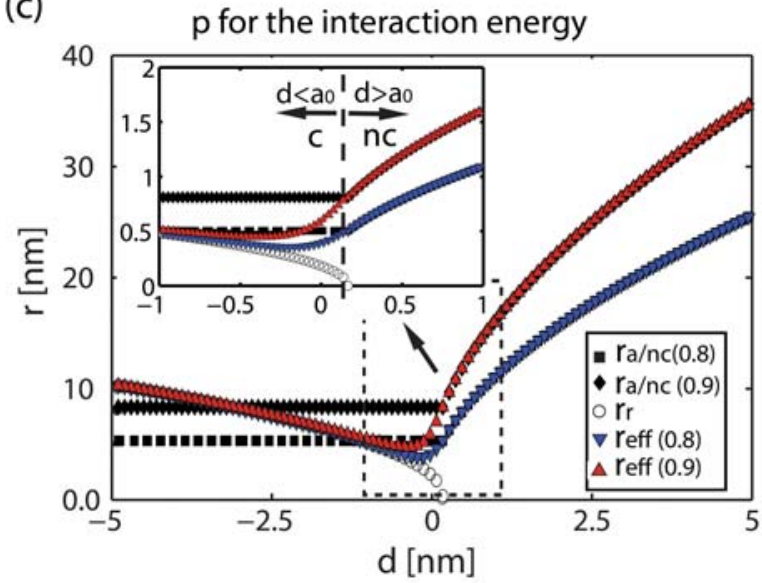

(b)

$\delta$ : deformation

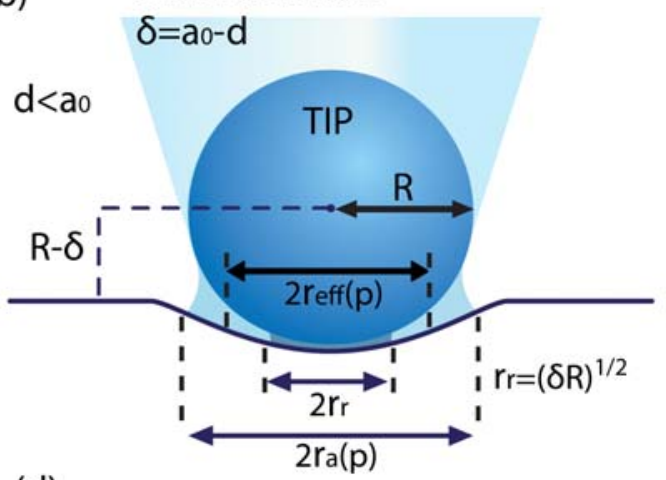

(d)

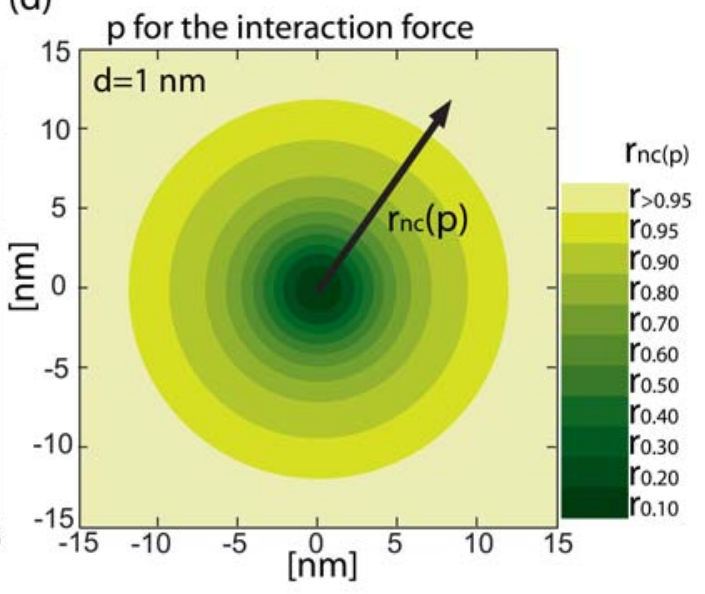

Figure 1. a) Scheme of the non-contact interaction area $S_{n c}$ for a tip in the proximity of a surface for the long range attractive forces $\left(d>a_{0}\right)$. The tip radius is termed $R$. The gradient shows how the effective radius $r_{n c}$ grows as larger fractions $p$ of the interaction are considered. This radius, and area, are thus termed $r_{n c}(p)$ and $S_{n c}(p)$ respectively. b) Scheme of effective radius $r_{a}(p)$ (attractive interactions) and $r_{r}$ (repulsive interactions) in the contact region where indentation occurs $\left(d<a_{0}\right)$. The effective value $r_{\text {eff }}$ is a combination of $r_{r}$ and $r_{a}(p)$. c) Radius of interaction $r$ versus distance $d$ for $r_{a / n c}(p)$ (squares and rhombuses), $r_{r}$ (outlined circles) and $r_{\text {eff }}(p)$ (triangles) for $p=0.8$ (red) and 0.9 (blue). Since $r_{a}(p)$ and $r_{n c}(p)$ are obtained from the same equations (Eqns. (1) and (2)) with the only difference being that for $r_{a}(p) d=a_{0}$, both are shown with the same markers as $r_{a / n c}(p)$ (squares and rhombuses). In the non-contact region, $n c ; r_{\text {eff }}(p)=r_{a / n c}$ (p). d) Relationship between $r$ and $p$ for the long range interaction force. The vdW and DMT forces have been used to model the nc and c interaction areas throughout. A tip radius of $20 \mathrm{~nm}$ has been used to produce c) and d). 


\section{Model}

\subsection{The static area of interaction}

We calculate the areas of interaction using the conservative long range van der Waals (vdW) and the short range repulsive forces. For the attractive forces, consider the AFM tip as a nanoscale sphere of radius $\mathrm{R}$ interacting with an infinite surface at a distance $\mathrm{d}$ (Fig. 1a). For this we use the Hamaker approach[24] where the long range vdW interaction between the tip and the surface is a triple volume integral for the atom-atom interaction. Here, in principle, all the atoms in the sphere are interacting with all the atoms in the infinite plane surface. Nevertheless, the actual interaction can be reduced to an arbitrary fraction of the total energy. We term this fraction $p$ (Fig. 1a) and write $r_{a}(p)$ and $r_{n c}(p)$ for the $c$ and $n c$ regions where the attractive force acts (Fig. 1b). In Fig. 1a, $r_{n c}(p)$ is schematically shown to grow with increasing $p\left(d>a_{0}\right)$. Here $a_{0}$ is an intermolecular distance which implies interpenetration between solid bodies cannot occur[25]. It can be shown [22, 26-27] (see supplementary for details) that for $r_{n c}(p)$

$$
\begin{aligned}
& r_{n c}(0.8)=0.83 R^{1 / 3} d^{3 / 5}+0.1 R \\
& r_{n c}(0.9)=1.12 R^{1 / 3} d^{3 / 5}+0.2 R
\end{aligned}
$$

where for the nc region $\left(d>a_{0}\right)$, $d$ is simply the tip-surface distance. For the c region $\left(d<a_{0}\right)$ Eqns. (1) and (2) become saturated (Fig. 1c), like the adhesive force [28], and provide the radius of the attractive component of the interaction energy in the contact region by simply putting $d=a_{0}$. This gives $r_{a}(p)$ (Fig. 1b). The relevance of the 0.8 and 0.9 values is that beyond these, $r_{n c}(p)$ rapidly and exponentially increases with $p$ [26-27]. A closed form for $r_{n c}(p)$ can be found by considering the force of interaction instead of 
the energy. Still, this expression remains explicit (see supplementary for details). The above takes care of $S_{c}\left(d<a_{0}\right)$ and $S_{n c}\left(d>a_{0}\right)$ for the attractive forces. [29]

For the repulsive nc forces $\left(d<a_{0}\right)$, we use the Derjaguin-Muller-Toporov (DMT) model of contact mechanics [28] that predicts a contact radius

$$
r_{r}=(R \delta)^{1 / 2} \quad \mathrm{~d}<\mathrm{a}_{0} \quad \text { (repulsive forces) }
$$

where $\delta$ is the deformation in the contact (Fig. 1b). Thus, when the tip and the surface are in mechanical contact $\left(d<a_{0}\right)$, the effective radius due to the attractive and the repulsive interaction forces are the two contributions to a net value of effective radius $r_{\text {eff }}(p)$. The superposition of the attractive and the repulsive forces in the contact region producing $r_{\text {eff }}(p)$ is schematically shown in Fig. 1b. Mathematically, this can be defined as

$$
r_{\text {eff }}(p)=\frac{r_{a}(p) F_{a}+r_{r} F_{r}}{F_{a}+F_{r}}
$$

where $F_{a}$ and $F_{r}$ are the attractive and the repulsive forces in the contact respectively, with $F_{a}$ derived from vdWs calculations $[24]$ and $F_{r}$ from the DMT model $[28,30]$. In Fig. 1c, $r_{a / n c}(p), r_{r}$ and $r_{e f f}(p)$ are plotted as a function of $d$ for $p=0.8$ and 0.9 and for $\mathrm{R}=20 \mathrm{~nm}$ by using Eqn. (4) and $\mathrm{F}_{\mathrm{a}}$ and $\mathrm{F}_{\mathrm{r}}$ as defined above. Furthermore, the relationships for the interaction energy (Eqns. (1) and (2)) have been used to obtain $r_{a / n c}$ (p). In the nc region, $r_{n c}(p)$ and $r_{\text {eff }}(p)$ are equal since only the nc forces act. Finally, Fig. 1d shows the relationship between $r_{n c}$ and $p$ for the interaction force at $d=1 n m$ and for $\mathrm{R}=20 \mathrm{~nm}$. The decreasing color gradient shows an increasing effective radius $\mathrm{r}_{\mathrm{nc}}$ (and $r_{\text {eff }}$ since they coincide there) with increasing $p$. Note that even at this small separation $(\mathrm{d}=1 \mathrm{~nm})$ the diameter of $\mathrm{S}_{\mathrm{nc}}$ for $\mathrm{p}=0.8$ is already $\sim 15 \mathrm{~nm}$. 

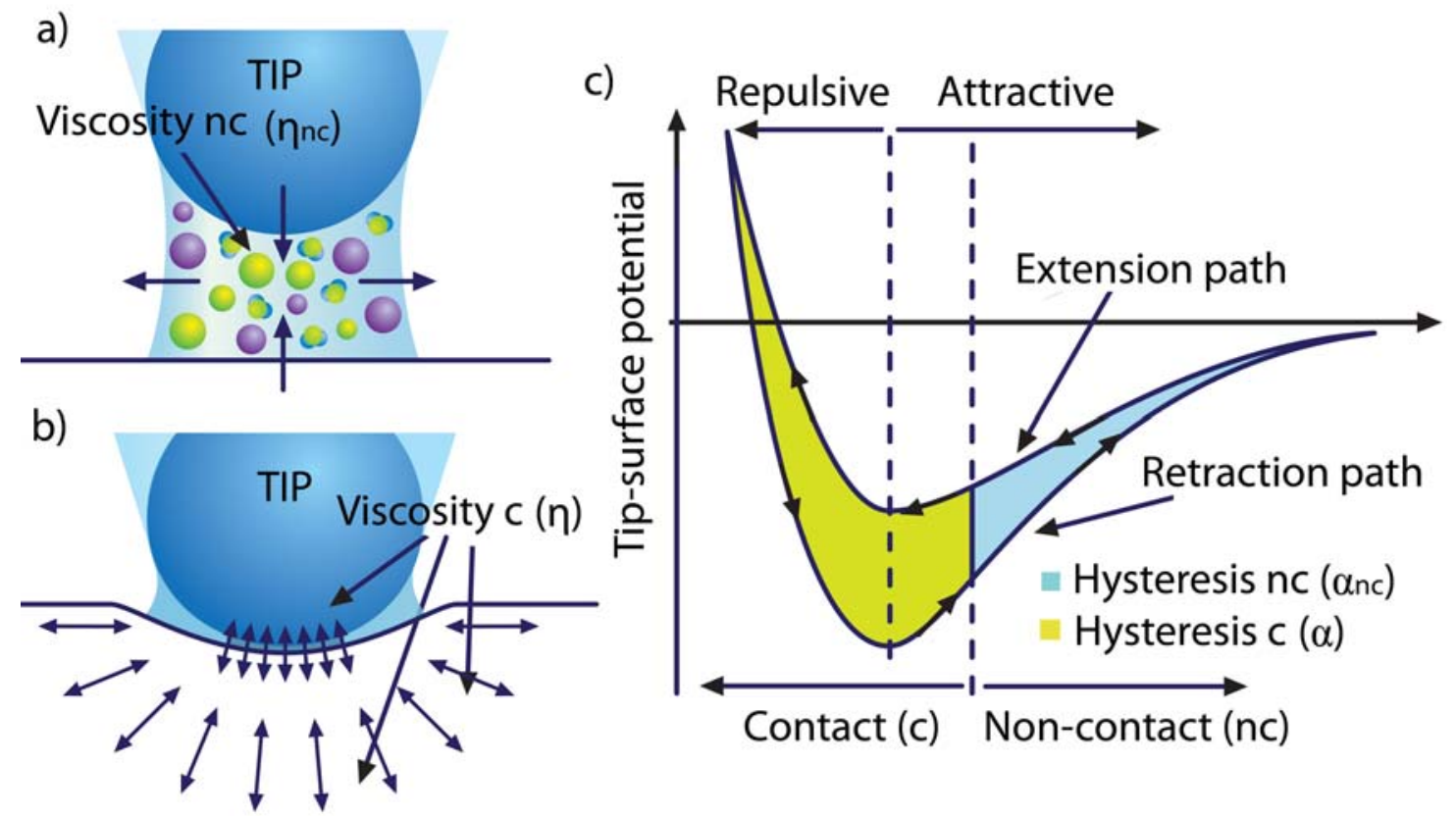

Figure 2. Schemes depicting the origins of viscosity in a) the nc region and b) the $\mathrm{c}$ region. c) Hysteresis processes are characterized by an increment in force, or potential energy as depicted, on retraction, as the tip moves away from the surface, as compared to extension. The scheme of the extension/retraction path is indicated by arrows. See text for details.

\subsection{The dissipative forces}

The second step involves modeling the dissipative interactions. Hysteresis and viscous processes in both the $\mathrm{nc}$ and the c regions form the four elementary ways in which energy can be dissipated in dynamic AFM. The viscosity in the nc region is related to; molecular displacement with tip motion, nanoscale confinement in the volume that lies in between the tip and the surface, general thermal excitation caused by atom collisions and breaking or disturbing chemical bonds either as the tip approaches or retracts (Fig. 2a). Here $\eta$ is the chosen symbol for viscosity. Physically, and from geometrical considerations, this implies a nc viscous force $F_{\eta_{\text {nc }}}$ which increases with decreasing separation and increasing radius of interaction $r_{n c}$ and velocity $\dot{z}$. The force in Eqn. (5) takes these factors into account respectively. The final expressions give

$$
F_{\eta_{n c}}=-\eta_{n c} r_{n c} \dot{z} \quad \mathrm{~d}>\mathrm{a}_{0}
$$




$$
F_{\eta}=-\eta(R \delta)^{1 / 2} \dot{z} \quad \mathrm{~d}<\mathrm{a}_{0}
$$

where $\eta_{\mathrm{nc}}$ is a exponential function of $\mathrm{d}$ (see supplementary for details).

The viscosity in the c region is modelled with the Voigt model as in previous studies $[19,23]$ and is given by Eqn. (6), where $\eta$ is a viscosity constant which is dependent on material properties [19]. A scheme representing the viscosity in this region is shown in Fig. 2b. The term may account for the local rearrangement and displacement of atoms, atomic reorientation and thermal excitation due to the relative motion between the atoms in the bodies in contact and the possible viscoelastic deformation of the tipsurface asperities [31].

The other elementary inelastic interaction is hysteresis [19], and this can be modelled also in the nc and the c regions as

$$
\begin{array}{ll}
F_{\alpha, n c}=-\alpha_{n c} \frac{R H}{6 d^{2}} & \mathrm{~d}>\mathrm{a}_{0} \\
F_{\alpha}=-\alpha \frac{R H}{6 a_{0}^{2}} & \mathrm{~d}<\mathrm{a}_{0}
\end{array}
$$

where $\alpha_{\mathrm{nc}}$ and $\alpha>0$ for retraction and zero for extension (Fig. 2c). Extension and retraction refer to the tip approaching and retracting from the surface respectively. The $\alpha$ coefficients in (7) and (8) control the strength of the hysteresis in the nc and c regions respectively. $\mathrm{RH}$ is the term accounting for the tip radius and the tip surface chemical properties through $\mathrm{R}$ and the Hamaker constant $\mathrm{H}$ respectively [24]. The hysteresis effects are related to the chemical affinity and reactivity between the tip and surface and 
the relative ease with which bonds (be they induced dipoles, hydrogen bonding or any other) form, as the tip approaches the sample near the contact region $\left(\mathrm{d} \sim \mathrm{a}_{0}\right)$ and break, as the tip retracts.

\subsection{The dynamic area of interaction}

Each of the four inelastic forces can now be linked to its respective interaction area as given by the expressions in Eqns. (1) to (3). For the viscosity and hysteresis in the nc (Eqns. (5) and (7)) we take $r_{n c}(0.8)$ where $p=0.8$ has been assumed. For the viscosity and hysteresis in the c (Eqns. (6) and (8)) we take $r_{r}$ and $r_{a}(0.8)$ respectively. Then, for each of the dissipative processes, the effective area of interaction per cycle in the dynamic mode $<\mathrm{S}>$ can be found as $[22,26]$

$$
<S>=\frac{1}{<F>} \sum_{i=1}^{i=n} S_{i}\left|F_{i}\right|
$$

where i stands for the instantaneous value of both the $\mathrm{S}$ and the $\mathrm{F}$ terms for a given tipsurface distance $d$ and $n$ refers to the points per cycle. The value of $<\mathrm{S}>$ converges rapidly for $n>128[22,26]$. In the simulations we have taken $n=256$. Furthermore

$$
<F>=\sum_{i=1}^{i=n}\left|F_{i}\right|
$$

Thus $<\mathrm{S}>$ as given in Eqn. (9) will control the resolution of a given dissipative process while the resolution of all dissipative process, i.e. phase contrast, will be controlled by the effective area of interaction of the sum of each process (see supplementary for details). The mean areal density value $<\rho>$ for the energy dissipated in the effective area $<\mathrm{S}>$ can now be defined as 
$<\rho>=<E>/<S>$

where for the sum of the processes, $<\mathrm{E}>$ is the mean energy dissipated per cycle in the tip-surface interaction and can be experimentally calculated from the phase lag $[16,19$, 32]. Nevertheless, we are interested here in $\langle\rho\rangle$ and, more particularly, in the relationship between $<\rho>$ and $<\mathrm{S}>$. These two parameters can be used to define a figure of merit $<\mathrm{M}>$

$$
<M>=\frac{<\rho>}{<S>}
$$

where high values of $<\mathrm{M}>$ imply highly localized energy interactions through high energy density $<\rho>$, and high resolution, i.e. small $<\mathrm{S}>$, or a combination of both. We use a point mass model where the cantilever stiffness $\mathrm{k}$ is modelled as a spring and the tip as a sphere of radius $\mathrm{R}$ and effective mass $\mathrm{m}$. It has been previously shown that this model is a good approximation to the phenomena in ambient conditions where the $\mathrm{Q}$ factor is high $\left(\mathrm{Q} \sim 10^{2}-10^{3}\right)[33]$. We have implemented the model in Matlab with the use of a standard fourth order Runge Kutta algorithm [34]. Besides the four dissipative forces above, the conservative long range attractive ( $\mathrm{vdW}$ ) and short range repulsive (DMT) forces have been added to the equation of motion [33, 35-36]. The long range processes, in the attractive regime, are discussed first. 


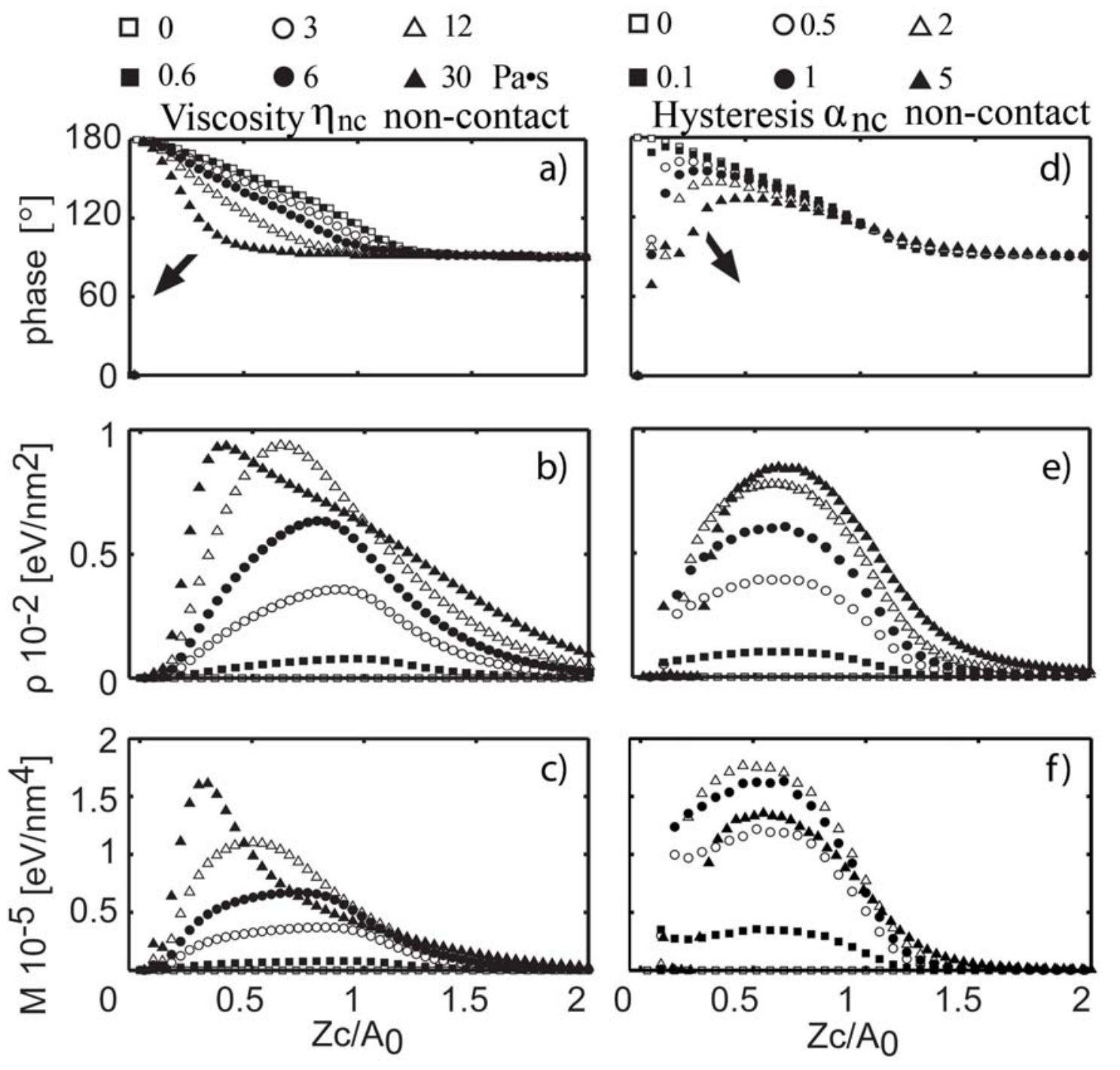

Figure 3. Simulations of the response of the phase shift, energy density $<\rho>$ and $<M>$ as a function of normalized separation $z_{\mathrm{c}} / \mathrm{A}_{0}$ with increasing dissipative non-contact coefficients $\eta_{\mathrm{nc}}$ and $\alpha_{n c}$ a,d) Phase b,e) density $<\rho>$ and $\left.\mathrm{c}, \mathrm{f}\right)<\mathrm{M}>$ for viscosity $\eta_{\mathrm{nc}}$ and hysteresis $\alpha_{\mathrm{nc}}$ respectively. The evolution of the phase shift for increasing $\eta_{\mathrm{nc}}$ and $\alpha_{\mathrm{nc}}$ is that indicated by the direction of the black arrows in a) and d) respectively. The maxima in $<\mathrm{E}>$ are $\sim 10 \mathrm{eV}$ for $\eta_{\mathrm{nc}}=30 \mathrm{~Pa} \cdot \mathrm{s}$ and $\sim 6 \mathrm{eV}$ for $\alpha_{\mathrm{nc}}=5$ (see supplementary Fig. S1 for details) and always increase with $\eta_{\mathrm{nc}}$ and $\alpha_{\mathrm{nc}}$ respectively. The simulation parameters are: $\mathrm{f}=\mathrm{f}_{0}=300 \mathrm{kHz}$ (drive and natural frequency), $\mathrm{k}=40 \mathrm{~N} / \mathrm{m}, \mathrm{R}=20 \mathrm{~nm}, \mathrm{Q}=500, \gamma=30 \mathrm{~mJ}$ (surface energy), $\mathrm{E}=10 \mathrm{GPa}$ (elastic modulus of the surface), $\mathrm{E}_{\mathrm{t}}=120 \mathrm{GPa}$ (elastic modulus of the tip) and $\mathrm{A}_{0}=5 \mathrm{~nm}$. See text for details. 


\section{Results and discussion}

In Fig. 3 the two elementary nc processes have been implemented separately and shown in the left and right columns respectively. Throughout the discussion, phase shifts imply differences between zero dissipation and non-zero dissipation since this is the mechanism behind phase contrast; see the arrows showing the tendency of the phase shift with increasing values of the dissipative coefficients (Figs. 3 and 4). In Fig. 3 all data has been acquired in the nc region (see supplementary for details). The characteristic behaviour of the phase shift with increasing $\eta_{\mathrm{nc}}$ is shown in Fig. 3a. Here, the most important characteristic is that there is maxima at intermediate $\mathrm{z}_{\mathrm{c}} / \mathrm{A}_{0} \sim 0.5$ to larger separations $\mathrm{z}_{\mathrm{d}} / \mathrm{A}_{0}>0.5$. Moreover, the phase shift, $<\rho>$ (Fig. 3b) and $<\mathrm{M}>$ (Fig. 3c) initially have maxima at intermediate $\mathrm{z}_{\mathrm{c}} / \mathrm{A}_{0} \sim 0.5$ to larger $\mathrm{z}_{\mathrm{c}} / \mathrm{A}_{0}>0.5$ separations but tend towards the smaller separations $z_{\mathcal{C}} / A_{0}<0.5$ with increasing $\eta_{\mathrm{nc}}$. A different pattern is followed by $<\mathrm{E}>$ (see supplementary Fig. S1) since, the maxima, remain always at the larger separations $\mathrm{z}_{\mathrm{c}} / \mathrm{A}_{0}>0.5$ and follow the opposite direction with increasing $\eta_{\mathrm{nc}}$. Thus, it is possible to obtain larger values of phase contrast, $<\rho>$ and $<M>$ at lower values of $<\mathrm{E}>$. Also note that, the maxima in phase shift, $<\rho>,<\mathrm{M}>$ and $<\mathrm{E}>$ increase with $\eta_{\mathrm{nc}}$. This implies that energy localization increases with increasing $\eta_{\mathrm{nc}}$. For long range hysteresis (Fig. 3d), the behaviour of the phase shift is radically different to that displayed by long range viscosity. Here, the larger phase shifts are expected at the smaller separations $\mathrm{z}_{\mathrm{c}} / \mathrm{A}_{0}<<0.5$ even if these still increase with $\alpha_{\mathrm{nc}}$. Furthermore, the maxima for both $<\rho>$ (Fig. 3e) and $<\mathrm{M}>$ (Fig. 3f) always fall at the larger separations $\mathrm{Z}_{\mathrm{c}} / \mathrm{A}_{0}>0.5$. Physically, this implies that large values of $<\rho>$ and $<\mathrm{M}>$ do not follow large values of phase contrast. Additionally, while $<\rho>$ monotonically increases with $\alpha_{\mathrm{nc}}$, $<\mathrm{M}>$ does not. The maxima in $<\mathrm{E}>$ for long range hysteresis lie at intermediate to large 
separations $\mathrm{z}_{\mathrm{c}} / \mathrm{A}_{0}>0.5$ and increase with $\alpha_{\mathrm{nc}}$ (see supplementary for details). The repulsive interactions are discussed next.
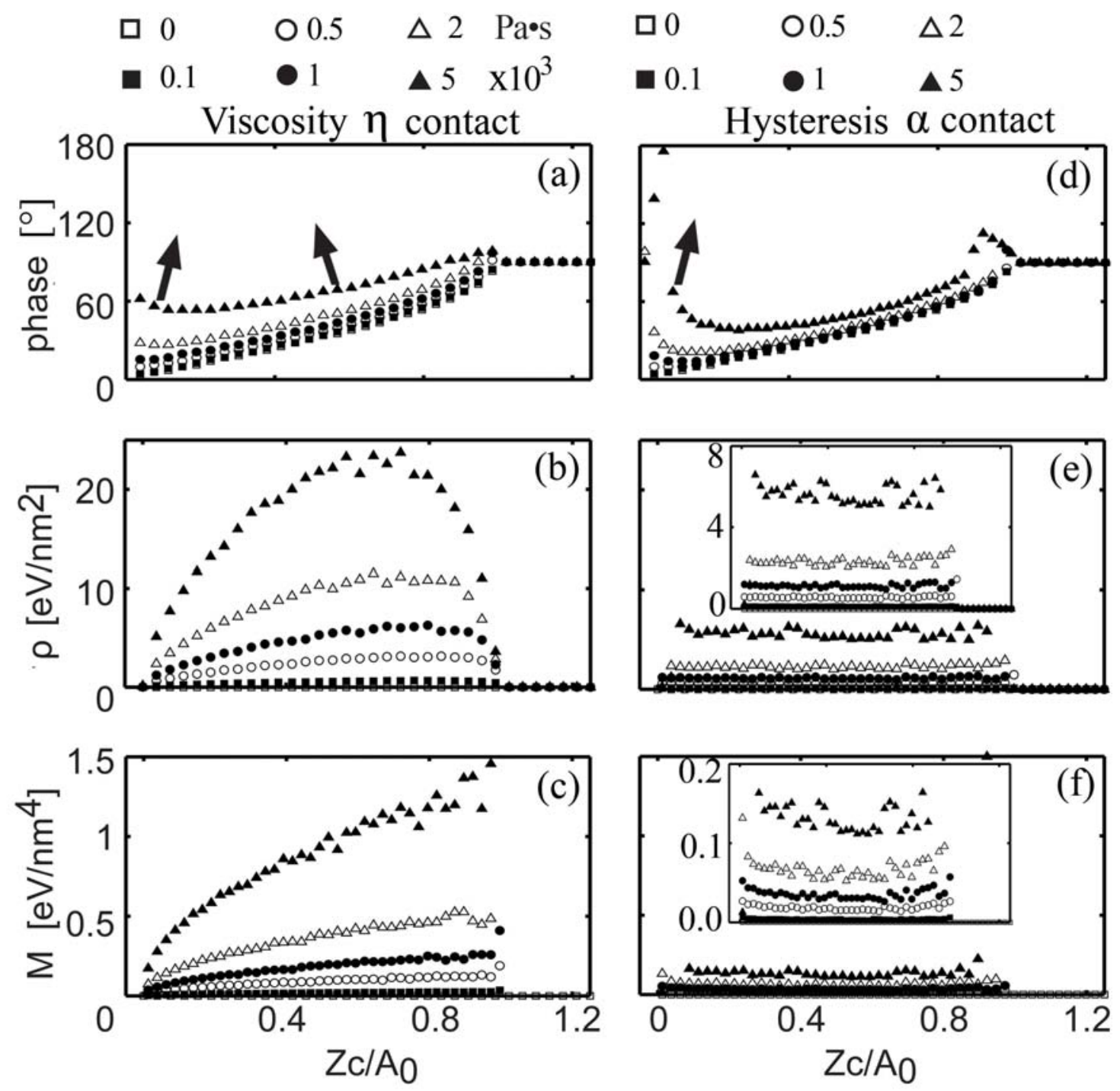

Figure 4. Simulations of the response of the phase shift, energy density $\rho$ and $<M>$ as a function of normalized separation $z_{\mathrm{c}} / \mathrm{A}_{0}$ and increasing contact coefficients $\eta$ and $\alpha$. a,d) Phase b,e) density $<\rho>$ and $\mathrm{c}$,f) $<\mathrm{M}>$ for viscosity $\eta$ and hysteresis $\alpha$ respectively. The evolution of the phase shift for increasing $\eta$ and $\alpha$ is that indicated by the direction of the black arrows in a) and d) respectively. The maxima in $\angle \mathrm{E}>$ are $\sim 550 \mathrm{eV}$ for $\eta=5 \times 10^{3} \mathrm{~Pa} \cdot \mathrm{s}$ and $\sim 250 \mathrm{eV}$ for $\alpha=5$ (see supplementary Fig. S1 for details) and always increase with $\eta$ and $\alpha$ respectively. The simulation parameters are as in Fig. 3 but with $A_{0}=40 \mathrm{~nm}$. The insets are zoomed of e) and f) respectively. See text for details. 
In Fig. 4a two arrows indicate the tendency of the phase shift with increasing $\eta$. Note that short range viscosity affects phase shifts at large $\left(\mathrm{z}_{\mathrm{c}} / \mathrm{A}_{0}>0.5\right)$ and small $\left(\mathrm{z}_{\mathrm{c}} / \mathrm{A}_{0}<0.5\right)$ separations. Nevertheless, while the larger values of phase shift are also expected for the smallest separation $\mathrm{z}_{\mathrm{c}} / \mathrm{A}_{0}<0.5$, both $<\rho>$ (Fig. 4b) and $<\mathrm{M}>$ (Fig. 4c) have maxima at the larger separations $z_{c} / A_{0}>0.5$. This implies highly localized energy dissipation at the larger separations $\mathrm{z}_{\mathrm{c}} / \mathrm{A}_{0}>0.5$. It is precisely at this region that tapping mode $A F M$ is typically operated, [37-38] implying highly localized energy dissipation without necessarily optimizing phase contrast. Furthermore, Figs. $4 b-c$ show that both $<\rho>$ and $<\mathrm{M}>$ monotonically increase with $\eta$. Additionally, also for short range hysteresis processes (Fig. 4d), the phase contrast is mostly affected at the smaller separations $\mathrm{z}_{\mathrm{c}} / \mathrm{A}_{0}<<0.5$. Here, both $<\rho>$ and $\mathrm{M}$ (Figs. $4 \mathrm{e}$ and $4 \mathrm{f}$ ) increase monotonically with $\alpha$, following the trend of $<\mathrm{E}>$ (see supplementary for details). Note here, that neither $<\rho>$ nor $<\mathrm{M}>$ show any dependency on $\mathrm{z}_{\mathrm{c}} / \mathrm{A}_{0}$. Finally, also for this dissipative process, maxima in phase contrast do not correspond with maxima in $<\rho>,<\mathrm{M}>$ or $<\mathrm{E}>$.

\section{Conclusions}

In summary, we have defined a figure of merit $<\mathrm{M}>$ that indicates how localized energy dissipation processes are in dynamic nanoscale interactions. We have shown that neither the amount of energy dissipated in the interaction nor the phase contrast are sufficient 
criteria to establish whether the interaction is highly localized. That is, while the interaction might involve several hundreds of eVs, these might affect a wider area involving hundreds of atoms or molecules. On the other hand, a few eVs dissipated in the interaction might involve only a few tens of atoms. M accounts for these differences in the extent of the interactions. While $M$ and the energy per unit area have been calculated for four elementary dissipative processes, other more specific phenomena might occur in nanoscale dissipative processes. In this respect the method here presented can be readily implemented to account for those. Finally, we have shown that that large coefficients of viscosity and hysteresis do not directly result in phase contrast. These results should provide insight into energy dissipation processes in nanoscale dynamics.

The art figures have been created by graphic designer Maritsa Kissamitaki.

[1] Zhang L and Webster T J 2009 Nano Today 4 66-80

[2] Brydson R M, Hammond C, Mowbray D, Gibbs M R J, Todd I, Grell M, Hamley I W, Geoghegan M, Jones R A L and Leggett G J 2005 Nanoscale Science and Technology (Chichester: Wiley)

[3] Boehm H P, Clauss A, Fischer G O and Hofmann U 1962 Zeitschrift für anorganische und allgemeine Chemie 316 119-27

[4] Boehm H P, Setton R and Stumpp E 1994 Pure and Applied Chemistry 66 1893-901

[5] Geim A K and Novoselov K S 2007 Nature Materials 6

[6] Zhao X, Liu Y, Inoue S, Suzuki T, Jones R O and Andol Y 204 Physical Review Letters 92125502

[7] Collins F S, Green E D, E. G A and Guyer M S 2003 Nature 422 835-47

[8] Voet D and Voet J 1995 Biochemistry: John Wiley and Sons)

[9] Simmel F C and Dittmer W U 2005 Small $1284-99$

[10] Piner R D, Zhu J, Xu F, Hong and Mirkin A C 1999 Science 283 661-3

[11] Bustamante C and Keller D 1995 Physics today 48 33-8

[12] Bhushan B, Israelachvili J N and Landman U 1995 Nature 374 607-16

[13] Binnig G, Quate C F and Gerber C 1986 Physical Review Letters 56 930-3

[14] Martin Y, Williams C C and Wickramasinghe H K 1987 Journal of Applied Physics 61 4723-9 
[15] Albrecht T R, Grutter P, Horne D and Rugar D 1991 Journal of Applied Physics 69 668-73

[16] Cleveland J P, Anczykowski B, Schmid A E and Elings V B 1998 Applied Physics Letters 72 2613-5

[17] Melcher J, Carrasco C, Xu X, Carrascosa J L, Gomez-Herrero J, de Pablo P J and Raman A 2009 Origins of phase contrast in the atomic force microscope in liquids. In: Proceedings of the National Academy of Sciences of the United States of America, pp 13655-60

[18] Wang H, Djurisica A B, Chan W K and Xie M H 2005 Applied Surface Science 252 1092-100

[19] Garcia R, Gómez C J, Martinez N F, Patil S, Dietz C and Magerle R 2006 Physical Review Letters 97 016103-4

[20] Voïtchovsky K, Kuna J J, Antoranz Contera S, Tosatti E and Stellacci F 2010 Nature Nanotechnology 5401 - 5

[21] Martinez N and Garcia R 2006 Nanotechnology 17 S167-S72

[22] Santos S and Thomson N H 2011 Applied Physics Letters 98 013101-3

[23] Tamayo J and Garcia R 1996 Langmuir 12 4430-5

[24] Hamaker H C 1937 Physica 4 1058-72

[25] Israelachvili J 1991 Intermolecular \& Surface Forces: Academic Press)

[26] Santos S 2011 Dynamic Atomic Force Microscopy and applications in biomolecular imaging. In: Structural Molecular Biology, (Leeds: University of Leeds)

[27] Santos S, Billingsley D J, Bonass W A and Thomson N H The double-helix of single DNA molecules In: Unpublised,

[28] Derjaguin B V, Muller V and Toporov Y 1975 Journal of Colloid and Interface Science 53 314-26

[29] Gleyzes P, Kuo P K and Boccara A C 1991 Applied Physics Letters 58 2989-91

[30] Fischer-Cripps A C 2007 Introduction to contact mechanics: Springer)

[31] Fischer-Cripps A C 2004 Nanoindentation: Springer)

[32] Proksch R and Yablon D G 2011 Loss tangent Imaging with amplitude modulation atomic force microscopy.

[33] Rodríguez T R and García R 2002 Applied Physics Letters 80 1646-8

[34] MATLAB R2008a and SIMULINK T M, Inc., Natick, Massachusetts, US.

[35] Garcia R and San Paulo A 1999 Physical Review B 60 4961-7

[36] Stark R, Schitter G and Stemmer A 2003 Physical Review B 68 0854011-5

[37] Santos S, Barcons V, Font J and Thomson N H 2010 Nanotechnology 21 225710-20

[38] Garcia R and Perez R 2002 Surface Science Reports 47 197-301 